\title{
A Rare Disease of Familial Chylomicronemia Syndrome in a 20 Days Infant Begum NNF ${ }^{1}$, Sarker $\mathrm{M}^{2}$
}

DOI: https://doi.org/10.3329/jafmc.v15i1.48658

\begin{abstract}
Familial chylomicronemia syndrome is a group of very rare genetic disorders. It is inherited as autosomal recessive disorder. Its estimated incidence is 1 in 1000000 populations, characterized by deficient activity of an enzyme lipoprotein lipase (LPL) or apoprotein C-II, resulting into severe fasting hypertriglyceridemia and massive accumulations of chylomicrons in plasma. LPL deficiency typically presents in childhood with failure to thrive, colicky abdominal pain, eruptive xanthomas, lipemiaretinalis, pancreatitis and hepatomegaly. We are reporting a rare case of familial chylomicronemia in a 20 days old child who was presented with pneumonia, his plasma incidentally found like milk during routine collection of his blood sample. As the child was only 20 days old with very high triglyceride level, so breast feeding continued and cocktail therapy with low dose Tablet Fenofibrate, Tablet Niacin, Tablet Atovastatin started after referral to paediatric cardiologist at 3 months of age.
\end{abstract}

Key-words: Familial chylomicronemia, Familial lipoprotein lipase deficiency, Apo-lipoprotein C-II deficiency, Milky blood.

\section{Introduction}

Lipid disorder refers to elevation of plasma cholesterol and/or triglyceride or low level of plasma HDL which can be primary or secondary ${ }^{1}$. Primary disorders are transmitted genetically and usually manifests from childhood. Secondary disorders contribute to the most casesof dyslipidemia in adults which are due to sedentary lifestyle with excessive intake of saturated fats, cholesterol. These secondary causes may include diabetes, hypothyroidism, alcohol, oral estrogens, renal disease, diuretics and $\mathrm{B}$ blockers, retinoic acid drugs, and atypical antipsychotics ${ }^{2}$.

Familial chylomicronemia syndrome (FCS) is a disorder of lipoprotein metabolism due to familial Lipoprotein Lipase (LPL) or apo-lipoprotein C-II deficiency (Apo C-II) or the presence of inhibitors to lipoprotein lipase ${ }^{3}$. The disease has been described in all races. To date, several hundred patients with LPLdeficiency have been described ${ }^{3}$. Several mutations in the LPL gene locatedon chromosome 8p22 have been identified with familial LPL deficiency. More than 50 missense and nonsense mutations have been identified. The majority of mutations are located on exons 3, 5 and 6 which are responsible for the catalytic coding region of the gene ${ }^{4}$. ApoC-II gene mutation has also been identified ${ }^{4}$. Other extremely rare genetic disorders can present with chylomicronemia with severe hypertriglyceridemia.
Examples of these are familial apo-AV deficiency, familial Lipase Maturation Factor 1 (LMF1) deficiency and familial GPIHBP1 (Glycosylphosphatidylinositol anchored high density lipoprotein binding) deficiency ${ }^{3}$.

FCS is the most dramatic example of severe hypertriglyceridemia. Almostallpatients withfasting triglyceride levels inexcess of $1000 \mathrm{mg} /$ dl $(11.36 \mathrm{mmol} / \mathrm{L})$ have $\mathrm{FCS}^{5}$. It manifests as eruptivexanthomas, acute pancreatitis, hepatomegaly, splenomegaly foam cell infiltration of bone marrow and lipemiaretinalis. These patients usually have lipemic plasma due to marked elevation oftriglyceride and chylomicron levels ${ }^{6}$.

\section{Case Report}

Mannaf, 3 months old male child first issue of consanguineous parents (1st degree cousins) delivered at term by normal vaginal delivery (NVD) with $3.3 \mathrm{~kg}$ birth weight. He reported to Combined Military Hospital Bogra at his 20 days of age with the history of fever, cough and respiratory distress. During his routine blood investigation they found his blood became milky in colour after few minutes of collection. So they referred the case to $\mathrm{CMH}$ Dhaka, where the case was investigated thoroughly. He was discovered to have very high triglycerides level $2680 \mathrm{mg} / \mathrm{dl}$, other investigation revealed hemoglobin $8.5 \mathrm{gm} / \mathrm{dl}$, total WBC count: $14000 / \mathrm{mm} 3$. Platelets count $345000 / \mathrm{mm} 3$, blood sugar $85 \mathrm{mg} /$ $\mathrm{dl}$, thyroid function was normal. At the age of 3 months the child was referred to paediatric cardiologist of $\mathrm{CMH}$ Dhaka, where investigations revealed serum cholesterol $590 \mathrm{mg} / \mathrm{dl}$, serum triglyceride 1048 mg/dl, LDL-C 345mg/dl, VLDL 310 mg/dl, HDL-C $45 \mathrm{mg} / \mathrm{dl}$. Further history revealed no history of hyperlipidaemia in parents. There is no history of sudden death, premature cardiovascular disease or recurrent pancreatitis in family. On examination the child was active, no dysmorphic faces, no skin xanthomatous eruption, no hepatoleomegaly, examination of cardiovascular system was normal including blood pressure. Eye examination was normal. Liver and kidney function tests were normal. ECG and Echocardiogram were normal.

He was treated initially by pediatrician and patient developed severe form of hepatic failure for the use of lipid lowering agent. During referral to pediatric cardiologist, as the age of the child was 3 months so breast feeding was continued and treatment started with Tablet Fenofibrate-20 mg/kg/day. We weekly investigated the liver and renal function. After a few months of continuation of this drug patient developed impaired liver function, his serum bilirubin was $3.3 \mathrm{mg} / \mathrm{dl}$, serum ALT $650 \mathrm{U} / \mathrm{L}$, Alkaline phosphates

1. Brig Gen Nurun Nahar Fatema Begum, SBP, MBBS, FCPS, FRCP, FACC, FSCAI, Independence Awardee, Professor \& Head, Department of Paediatrics, AFMC, Dhaka (Email: colfatema@hotmail.com) 2. Lt Col Mukta Sarker, MBBS, FCPS, Classified Specialist in Paediatrics, $\mathrm{CMH}$, Bogura. 
$425 \mathrm{U} / \mathrm{L}$, so the drug was discontinued. After improvement of his liver function a cocktail therapy with low dose Tablet Fenofibrate, Tablet Niacin, Tablet Atovastatin started. With this cocktail therapy it was found that no further deterioration of liver and renal function with gradual improving the level of lipid profile. Now his blood no more milky, he isphysically and neurologically healthy and his lipid profile normal. Follow up is ongoing at every six monthly interval.

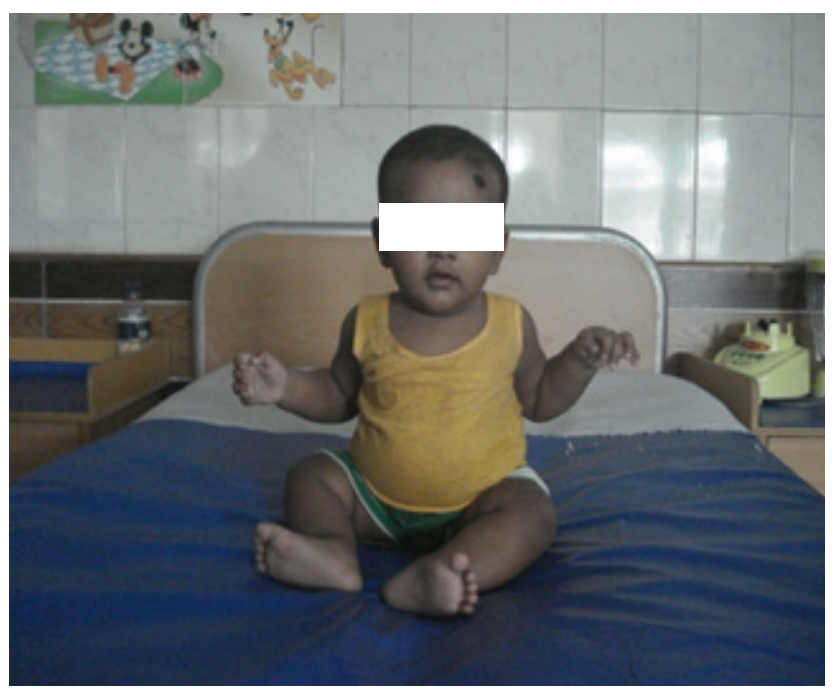

Figure-1: Mannaf

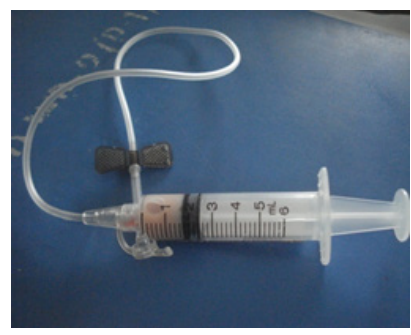

Figure-2a: Thick milky blood sample before starting treatment

\section{Discussion}

Hypertriglyceridemia is defined as having plasma triglyceride above the 95th percentile for age and sex. According to the National Cholesterol Education Program (NCEP), normal triglyceride level is $150 \mathrm{mg} / \mathrm{dl}(1.7 \mathrm{mmol} / /)^{7}$. Familial chylomicronemia is a very rare autosomal recessive (AR) disorder. It is caused by deficiency of extra hepatic lipoprotein lipase (LPL) or its cofactor apoproteinCII (Apo-ClI). Apo-Cll deficiency typicallyhas a later onset of symptoms and is often milder in appearance ${ }^{8}$. In comparison with patients with deficiency of Apo-Cll, patients with LPL deficiency present at an earlier age with more severe hypertriglyceridemia andlower tolerance to dietary fat ${ }^{1}$. This case was probably due to LPL deficiency as it manifested at 20 days ofage and triglyceride was markedly high.

FCS usually manifests in childhood, but $25 \%$ of cases manifested during infancy and are rarely manifest in thenewborn period9. In this case he was diagnosed at his 20 days of life. In India, several cases have been reportedin very young children aged between 20 and 60 days $^{9}$. Some presented with features of sepsis with systemic complications and acute renal failure with complete recovery ${ }^{4}$. But no data regarding infant with hyperchylomicronaemia available in Bangladesh. In the present report, an infant was described with FCS who was presented to us for some other reason like Pneumonia. Diagnosis of FCS was based on lipemic appearance of serum, serum triglycerides level of $2680 \mathrm{mg} / \mathrm{dl}$. Diagnosis is usually made by measurement of LPL immunoreactive mass in post heparin plasma and Apo-Cll levels by gel electrophoresis 10 . These tests could not be performed in this patient as these tests were not available in this setting. But clinical and biochemical picture pointed towards LPL deficiency.

FCS manifests during childhood with lipemic retinitis, hepatospleenomegaly, irritability and recurrent epigastric pain with increased risk of pancreatitis ${ }^{3}$. Patients with triglyceride levels above $2,000 \mathrm{mg} / \mathrm{dl}$ are more likely to present with xanthomas, and those with levels above $4,000 \mathrm{mg} / \mathrm{dl}$ may present with lipemiaretinalis (pale pink color to the retinal arteriolesand venules due to light scattering of the large chylomicrons) $)^{8}$. In this case triglyceride level was very high-2680 mg/dl, hemoglobin $(\mathrm{Hb})$ percentage was low $8.5 \mathrm{gm} / \mathrm{dl}$ (normal 13.6-19.6 g/dl), with no evidence ofhemolysis. He had no xanhoma, lipemic retinitis, hepatosplenomegaly, There was no family history of triglyceridemia. Lipid profile of parents was normal. Early diagnosis of FCS important to prevent complications such as acute and chronic pancreatitis andpancreatic necrosis, although pancreatic function often deteriorates very slowly ${ }^{1}$. The complication in thesechildren is due to increased amount of fat intake which causes recurrent bout of illness leading to formation ofcysts, hemorrhage and death.

Unfortunately, FCS resulting from deficiency in LPL or apo C-II is very difficult to treat with existingpharmacologic agents. The most effective treatment modality is severe dietarytriglyceride restriction. The recommended targets vary from less than $50 \mathrm{~g}$ per day, orunder $25 \%$ of total daily caloric intake, to less than $20 \mathrm{~g}$ per day, or under $15 \% \%^{9-11}$. But a significantly persistent hightriglyceride level necessitates pharmacological intervention. There has been a general reluctance to use drug therapy to treat lipid abnormalities in children; however, increasing evidence suggests effectiveness and short-term safety similar to those in adults $^{7}$. Recently, the American Heart Association has provided general recommendations for pharmacological management of high-risklipid abnormalities in children and adolescents. They defined high-risk lipid abnormalities as primary and secondary conditions associated with extreme lipid abnormalities or conditions underlying high risk of cardiovascular disease whereby the presence and severity of lipidabnormalities may further exacerbate that risk ${ }^{12-14}$.

The drugs studied and recommended for treating hypertriglyceridemia are fibric acid derivatives (e.g., Gemfibrozil, Fenofibrate). These have the effect on both raising HDL and lowering triglycerides. Main adverse effects observed were gastrointestinal upset together with an increased predisposition to cholelithiasis. Elevated liver transaminases and creatine kinase. There is risk of myopathy and rhabdomyolysis-especially if used with other agents, particularly statins ${ }^{7}$. Wheeler and colleagues performed a 6-months randomized cross-over trial of Bezafibrate in 14 children with familial hypercholesterolemia ${ }^{12}$. One patient had transient elevation in liver transaminase and one patient 
had elevation of alkaline phosphatase. The medication was well tolerated with no impact on growth or development. Other drugs, such as statins, were also studied and found to be effective in treating familial hypercholesterolemia but did not have much effect in lowering triglyceride level ${ }^{15}$. Niacin is not recommended becauseof limited available data ${ }^{16}$.

As the age of the child was 3 months, breast feeding was continuedand the child was treated with Tablet Fenofibrate. A few months after treatment patient developed impaired liver function, his serum Bilirubin $3.3 \mathrm{mg} / \mathrm{dl}$, serum ALT $650 \mathrm{U} / \mathrm{L}$, Alkaline phoshphatase $425 \mathrm{U} / \mathrm{L}$, so we discontinued this drug. After improvement of his liver function a cocktail therapy with lower dose of Tablet Fenofibrate, Tablet Niacin, Tablet Atorvastatin was started. With this cocktail therapy no further deterioration of liver and renal functionoccured with gradual correction of lipid profile. Literature and case reports on Familial chylomicronemia in infantile age group are scanty and there are no specific treatment guidelines in this age group. Recommendation for their use in young children needs further studies.

\section{Conclusion}

Familial chylomicronemia syndrome (FCS) is a disease of late childhood and adolescence; however, cases have been reported in infants and neonates. The syndrome presentation is heterogeneous in young age group. Early diagnosis and medical intervention by lipidlowering agents and dietary modification, at the time of diagnosis, can improve the prognosis and maintain a near normal lifestyle for affected children. This case treated with a special protocol of low dose Fenofibrate, Niacin and Atorvastatin with excellent outcome. However long-term studies are still needed to ensurethe safety and effectiveness of these agents on children.

\section{References}

1. Wani $K$, Rashid $M$, Alaqaband $M M$ et al. Familial ChylomicronemiaSyndrome (FCS) in a 10- Day- Old Neonate: A Case Report. Int J Pediatr 2015; 3(14):449-53.

2. Chaurasiya OS, Kumar L, Sethi RS. An Infant with Milky Blood: An Unusual but Treatable Case of Familial Hyperlipidemia. Ind J Clin Biochem 2013; 28(2):206-9.
3. Feoli-Fonseca JC, Levy E, Godard M et al. Familial lipoprotein lipase deficiency in infancy: Clinical, biochemical and molecular study. J Pediatr 1998; 133(3):417-23.

4. Santamarina F. The familial chylomicronemia syndrome. Endocrinol Metab Clin North Am 1998; 27(3):551-67.

5.Brunzell JD, Miller NE, Alaupovic P. Familial chylomicronemia due to a circulating inhibitor of lipoprotein lipase activity. J Lipid Res 1983; 24(1):12-9.

6. Guder GD, Narayanan H, Wisser H et al. Diagnostic Samples: From the Patient to the Laboratory: The Impact of Preanalytical Variables on the Quality of Laboratory Results. Wiley, New York, USA 2009:4.

7. De S, Halder S, Samanta S. A rare case of familial chylomicronemia in a two and half month old boy. IOSR-JDMS 2015; 14(10):48-50.

8. da Cruz EM, Ivy D, Jaggers J. Editors.Pediatric and Congenital Cardiology, Cardiac Surgery and Intensive Care. DOI 10.1007/978-14471-4619-3_55, \# Springer-Verlag London 2014.

9. Clauss SB, Kwiterovich PO. Genetic disorders of lipoprotein transport in children. Prog Pediatr Cardiol 2003; 17(2):123-33.

10. Homology of lipoprotein lipase to pancreatic lipase. Proc Natl Acad Sci USA 1986; 83(12):4185-9.

11. American Academy of Pediatrics: Committee on nutrition: Indications for cholesterol testing in children. Pediatrics 1989; 83:141-2.

12. Okura $Y$, Hayashi $K$, Shingu $T$ et al. Diagnostic evaluation of acute pancreatitis in two patients with hypertriglyceridemia. World $\mathrm{J}$ Gastroenterol 2004; 10:3691-5.

13. Clauss SB, Kwiterovich PO. Genetic disorders of lipoprotein transportin children. Progress in Pediatric Cardiology 2003; 17(2):123-33.

14. APOLIPOPROTEIN C-II, APOC2, Online Mendelian Inheritance in Man. Johns Hopkins University 2010.

15. Burnett JR, Hooper AM and Hegele RA. Familial Lipoprotein Lipase Deficiency. University of Washington, Seattle; 1993-2017. 1999 Oct 12 [updated 2017 Jun 22].

16. Ben-Avram CM, Ben-Zeev O, Lee TD et al. Homology of lipoprotein lipase to pancreatic lipase. Proc Natl Acad Sci USA 1986; 83(12):4185-9. 